\title{
TUNING PID CONTROLLERS USING HYBRID GENETIC AND NELDER-MEAD ALGORITHM
}

\author{
A. A. Lasheen ${ }^{1}$;A. M. El-Garhy' ${ }^{2}$; E. M. Saad ${ }^{3}$ and S.M.Eid ${ }^{4}$ \\ 1- Engineer of automatic control, Faculty of Eng. Helwan University, \\ Helwan, Egypt \\ 2, 3- Faculty of Eng. Helwan University, Egypt \\ 4- Faculty of Eng. Cairo University, Cairo, Egypt
}

(Received February 20, 2010 Accepted June 12, 2010)

\begin{abstract}
This Paper presents a hybrid genetic and Nelder-Mead algorithm (HGNMA) to determine the optimal proportional-integral-derivative (PID) controller parameters. PID controller exhibits good features, including easy implementation, stable convergence characteristic and good computational efficiency. The HGNMA algorithm is implemented in $M A T L A B$, the results obtained from this hybrid technique is compared with that obtained from Genetic Algorithm (GA) and the conventional PID which is tuned via the Ziegler-Nichols frequency response method. The proposed method is more efficient in improving the step response characteristics such as, reducing rise time, settling time and maximum overshoot.
\end{abstract}

KEYWORDS: PID controller parameter, Hybrid Genetic NelderMead (HGNMA).

\section{1- INTRODUCTION}

PID controllers have been widely used in most of control systems for a long time, due to their simplicity, clear functionality and ease of use, it provide robust and reliable performance for most systems. However, there is still a very important problem how to determine or tune the PID parameters [1], because these parameters have a great influence on the stability and the performance of the control system. Tuning a control loop is the adjustment of its control parameters (gain/ proportional band, integral gain/reset, derivative gain/rate) to the optimum values for the desired control response.

If the PID controller parameters are chosen incorrectly, the controlled process output can be unstable. The optimum behavior on a process change or setpoint change varies depending on the application, generally, stability of response is required and the process must not oscillate for any combination of process conditions and setpoints.

There are several ways to determine the parameters of a PID controller. One approach is to adjust the parameters manually until desired behavior is obtained, [2] but this approach has its disadvantages. The process can become unstable if it is tuned without caution and good knowledge about the system. It can also be very time consuming. Another approach is to make a mathematical model for the process based on some experiments; the Ziegler Nichols method is an experimental one that is widely used [2]. For a wide range of practical processes, this tuning approach works quite 
well. However, sometimes it dose not provide good tuning and is easy to produce surge and big overshoot, particularly for processes with serious non-linearity [3].

Intelligent optimization approaches have been used to improve PID tuning, such as genetic algorithm [4], neural network, fuzzy logic [5, 6] and practical swarm optimization [3], [8-10].

This paper has been organized as follows, Section 2 explains generalized model of PID controller. Section 3 introduces the hybrid genetic and nelder-mead algorithm, Section 4 presents the design of PID controller using (HGNMA). The proposed technique is simulated and applied on two higher order systems. The results are evaluated and given in Section 5. Finally, the conclusion is presented in Section 6.

\section{2- THE STANDARD PID CONFIGURATION}

The PID controller consists of proportional action, integral action and derivative action as shown in Fig. 1. In this configuration, the control signal $u(s)$ is the sum of three terms. Each of these terms is a function of the tracking error $E$ (s). Each of the terms works "independently" of the other and the general equation of it expressed by the following transfer function [1]:

$$
G_{c}(s)=K_{p}+K_{i} * 1 / s+K_{d} *{ }_{s} \ldots \ldots \ldots \ldots \text { (1) }
$$

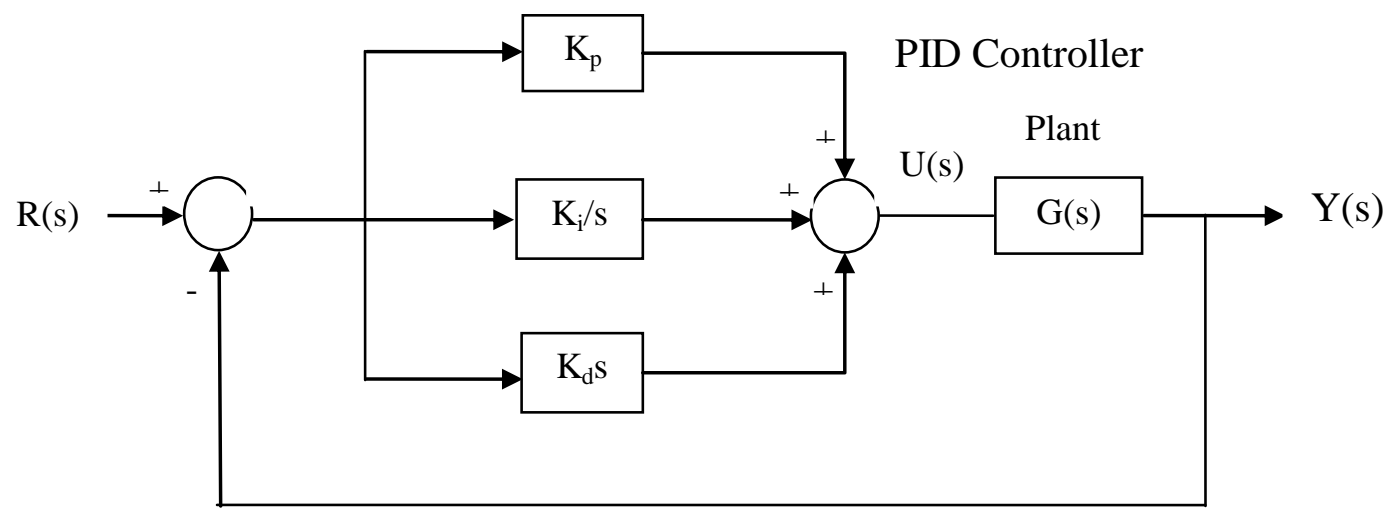

Fig. (1) Closed loop system with PID

Where;

$K_{p}:$ the proportional gain,

$K_{i}$ : the integral coefficient and

$K_{d}$ : the derivative coefficient.

The tuning of a PID controller is the process of selecting the controller parameters $K p, K i$ and $K d$ to meet given performance specifications as rise time, overshoot and settling time. To achieve this purpose, the controller compares the measured process output value $(Y)$ with the reference setpoint $(R)$ value. The difference or error signal $(E)$ is then processed to calculate the control signal for the manipulated process inputs, so the system output reaches the desired reference value, i.e the manipulated variable $(U)$ is adapted at the command of the controller based on its input data, which is the error $(E)[2]$. 


$$
\boldsymbol{E}=R-Y
$$

According to figure (1) and equation (1), the transfer function describes the feedback control system is:

$$
\frac{Y(s)}{R(s)}=\frac{G_{c}(s) G_{p}(s)}{\left[1+G_{c}(s) G_{p}(s)\right]}
$$

\section{3- THE HYBRID GENETIC ALGORITHM}

An effective optimization technique is dependent on its searching ability for global optimum solution and its accuracy. Genetic algorithm (GA) is a directed random search technique used to look for parameters that provide a good solution to a problem. It holds a population of solutions (often known as individuals or chromosomes). The separate parts of individuals are known as genes. Each individual is assigned a fitness value, which indicates the quality of the solution the chromosome represents. During the execution of a GA, population is continually replaced by new populations. The new populations are created by applying operators (crossover and mutation) to members of the Existing population. [7].

Genetic algorithms can be very powerful to find a global optimum area but are not very fast to solve local optimization problems. However, it is sometimes very difficult to find the minimum of a function using a genetic algorithm because bad solutions can be very near to the global optimum.

Local optimization techniques such as the Nelder-Mead Simplex have some common characteristics with genetic algorithm as they do not use the successive derivatives of the function and deals with a population of points instead of a single point. Furthermore, they are quite efficient to find a local optimum very quickly. In recent years, to enhance the global optimization searching ability of genetic algorithm, the genetic algorithm (GA) and the Nelder-mead method are both categorized into the primitive stage, that is, both of them are a direct search method without gradient information. Thus it has a fast searching ability and has been widely applied to improve conditions for complicated processes. [11-14]

There are many methods to utilize the idea of hybridizing local search techniques with the genetic algorithm. One method is to use GA to generate the individuals (solutions) for the new population and then apply the other method to improve this new population. The other idea of hybridizing process is to do some modifications in the genetic operations; selection, crossover, and mutation using local search methods. In this paper we use the first idea, where the GA generates the solutions for new population and then the Nelder-mead technique is used to improve the best solution which exists in the new population [12-14]

\section{4- IMPLEMENTATION OF HGNMA-BASED PID CONTROLLER}

The GA generates the solutions for the new population and then the Nelder-Mead technique is used to improve the best solution which exists in the new population. The steps involved in implementing a HGNM algorithm are as follows: 
1- Generate an initial, random population of individuals that consists of $\mathrm{N}$ chromosomes for a fixed size (KP, $\mathrm{Ki}, \mathrm{Kd}$ ranges declared).

2- Evaluate the fitness for each chromosome (to minimize integral square error).

3- Select the fittest members of the population.

4- Check for applying the Nelder-Mead algorithm, if no go to step 6.

5- Apply the Nelder-Mead algorithm on the best solution form the GA.

6- Check for optimality, if yes go to step 9.

7- Apply the GA operators (selection, crossover and mutation).

8- Apply Rotation Operator, go to step 2.

9- Check for overlap in the best solution, if no go to step 10.

10- Stop.

These steps can be illustrated in the flow chart Fig. (2).

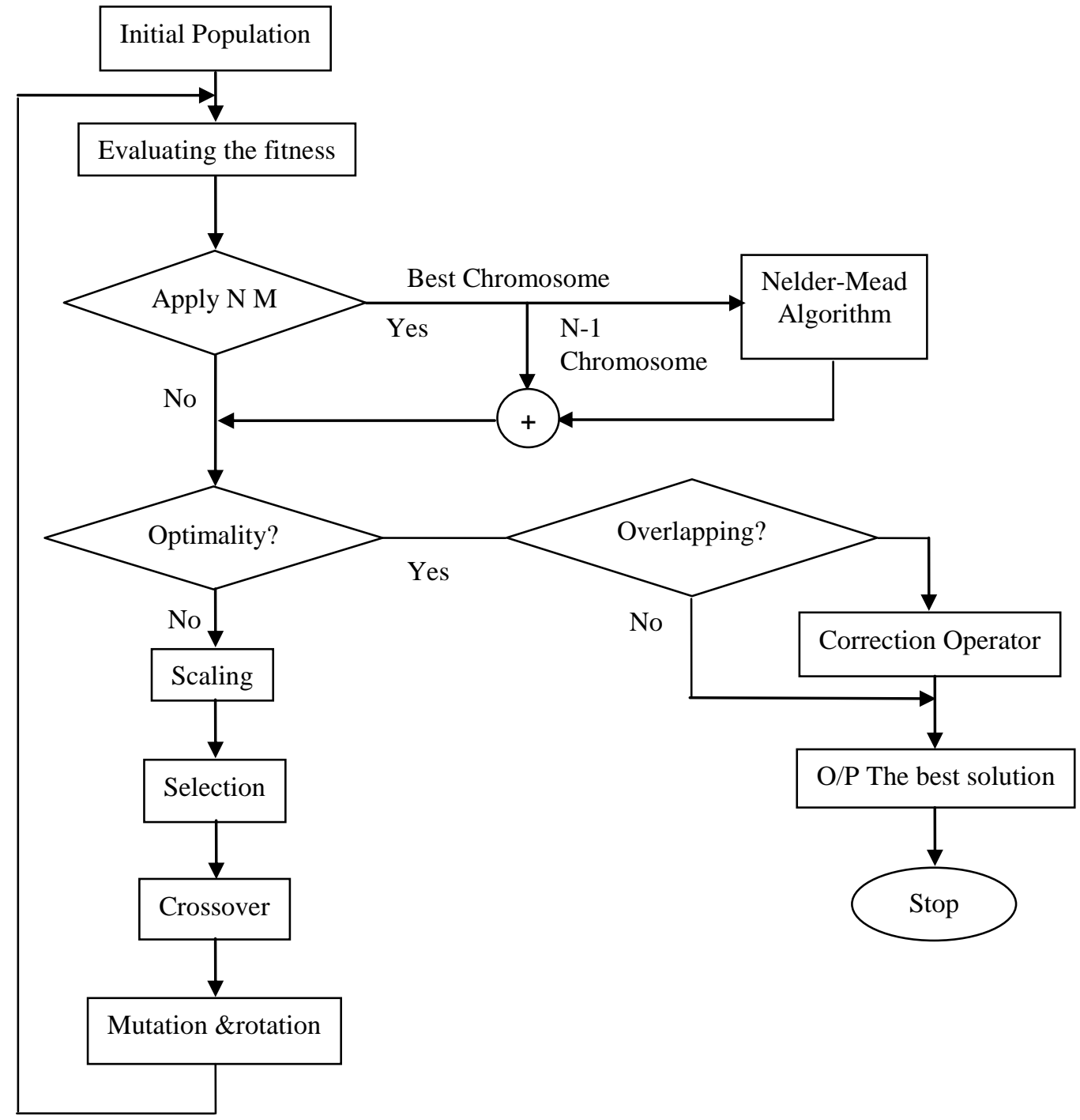

Fig. (2) Hybrid Genetic Algorithm 


\section{5- EXPERIMENTS AND RESULTS}

The HGNMA-based PID controller is applied to two systems the first one is a fourth order system, with the following transfer function:

$$
G_{1}(s)=\frac{1}{s^{4}+6 s^{3}+11 s^{2}+6 s}
$$

And the second one is a higher order system [2] with the following transfer function:

$$
G_{2}(s)=\frac{1}{(s+1)^{8}}
$$

Figure (1) illustrates the block diagram of a feedback control system with PID controller, and described by transfer function (3).

In order to compare the performance of different control algorithms, and to estimate the parameters of PID controller, the integral of the square of the error (ISE) is used as performance index [2] for the first system, indicated in (6).

$$
I S E=\int_{0}^{T} e^{2}(t) d t \ldots . .
$$

And for the second the follwing objective function is used as a performance index

$$
J=\sigma_{e}^{2}-\sigma_{u}^{2}
$$

Where:

$$
\sigma_{e}^{2}=\frac{1}{n} \sum_{1}^{n}\left(y_{s p}(i)-y(i)\right)^{2}
$$

$\sigma_{e}^{2}:$ is the variance of the controlerror $\mathrm{e}=\mathrm{y}_{\mathrm{sp}}-\mathrm{y}$;

$$
\sigma_{u}^{2}=\frac{1}{n} \sum_{1}^{n}(u(i)-\bar{u})^{2}
$$

$\sigma_{u}^{2}:$ variance of the control signal

$$
\bar{u}=\frac{1}{n} \sum_{1}^{n}(u(i)
$$

$$
\begin{aligned}
& \mathrm{W} \text { : is a weighting factor with range } \\
& 0 \leq w \leq 1
\end{aligned}
$$

The tuning of HGNMA-based PID controller' results are compared with those obtained from the GA and the Ziegler-Nichols method. These results are indicated in the tables (1) and (2) for the first system and tables (3) and (4) for the Second one. Figures (3-4) indicate the step response for the PID with the three Algorithms for the first system, and the second one. 
Table (1) PID controller steady state Responses

\begin{tabular}{|c|c|c|c|}
\hline Performance Specification & HGNMA & GA & Z-N \\
\hline Rise Time & 0.61 & 0.68 & 0.68 \\
\hline \% Overshoot & 24 & 38 & 58.1 \\
\hline Settling Time & 9.1 & 29.9 & 14.9 \\
\hline
\end{tabular}

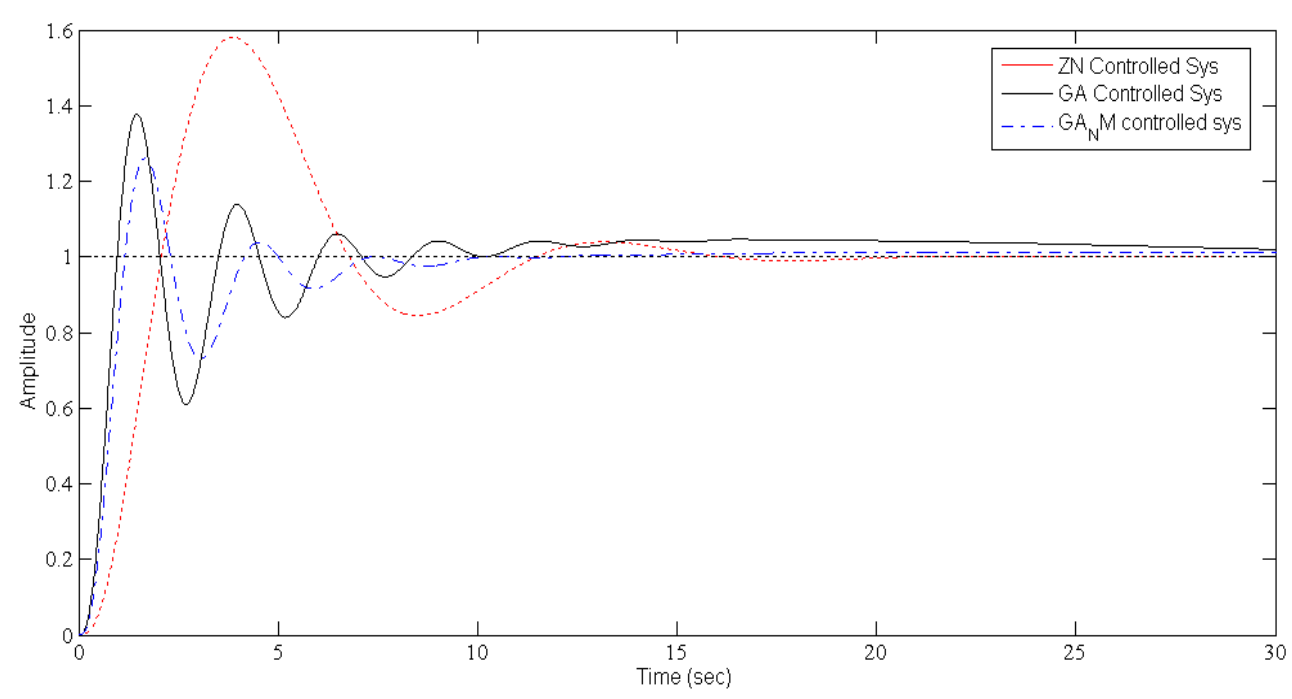

Fig. (3) Closed-loop step responses for the PID with the three Algorithms for the first system, $G_{1}(s)=\frac{1}{s^{4}+6 s^{3}+11 s^{2}+6 s}$

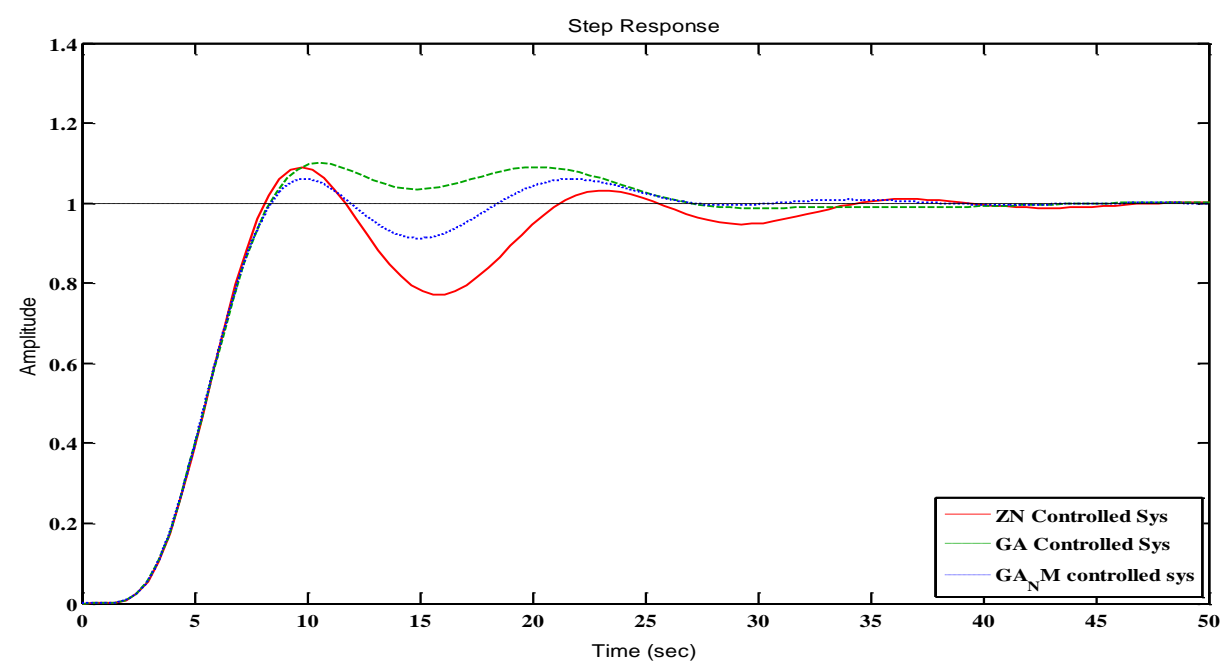

Fig. (4) Closed-loop step responses for the PID with the three Algorithms

$$
\text { For second system, } G_{2}(s)=\frac{1}{(s+1)^{8}}
$$



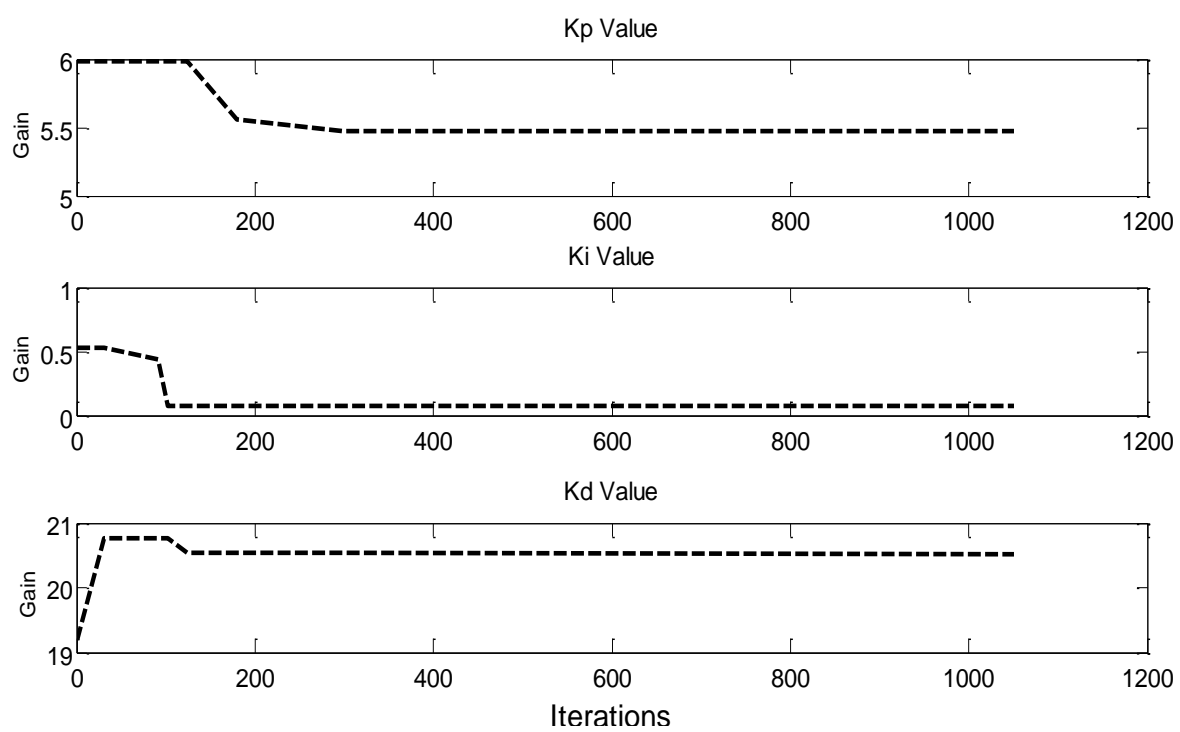

Fig. (5) Illustration of the HGNMA Converging through iterations

The parameters of the PID controller for the three approaches were being as indicated in tables (2) for the first system, and (4) for the second system.

Table (2) PID controller gains for the first system

\begin{tabular}{|c|c|c|c|}
\hline Controller Parameters & HGNMA & GA & Z-N \\
\hline $\mathrm{K}_{\mathrm{p}}$ & 5.47112 & 6.85821 & 6 \\
\hline $\mathrm{K}_{\mathrm{i}}$ & 0.08097 & 0.23002 & 1.91 \\
\hline $\mathrm{K}_{\mathrm{d}}$ & 20.51549 & 21.07064 & 4.74 \\
\hline
\end{tabular}

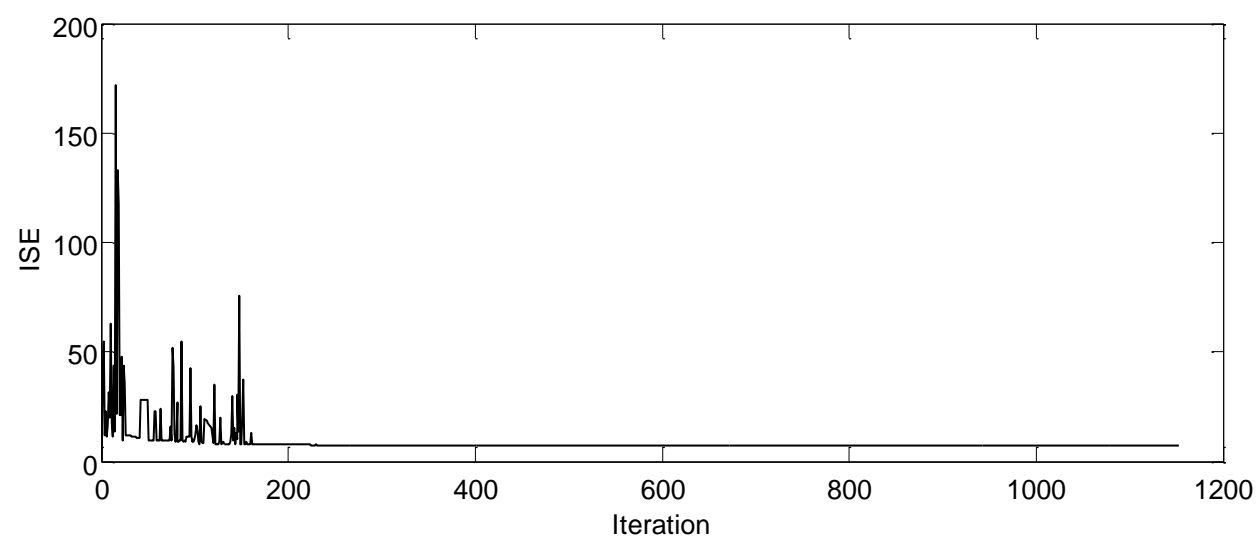

Fig. (6) Illustration of the HGNMA Converging through iterations. 
Table (3) PID controller steady state Responses for second system

\begin{tabular}{|l|c|c|c|}
\hline Performance Specification & HGNMA & GA & Z-N \\
\hline Rise Time & 4.09 & 4.22 & 4.03 \\
\hline \% Overshoot & 6 & 10 & 9.1 \\
\hline Settling Time & 24 & 25.4 & 32.7 \\
\hline
\end{tabular}

Table (4) PID controller gains for second system

\begin{tabular}{|c|c|c|c|}
\hline Controller Parameters & HGNMA & GA & Z-N \\
\hline $\mathrm{K}_{\mathrm{p}}$ & 0.95058 & 0.87039 & 1.13 \\
\hline $\mathrm{K}_{\mathrm{i}}$ & 0.18760 & 0.22427 & 0.14907 \\
\hline $\mathrm{K}_{\mathrm{d}}$ & 2.39918 & 2.42484 & 2.147 \\
\hline
\end{tabular}

\section{6- CONCLUSION}

The paper presented a design of PID controller using Z-N technique, GA and hybrid genetic and Nelder-Mead algorithm HGNMA for higher order system. The simulation results for the two systems confirm that the tuned HGNMA controller can provide better performance comparing with the Ziegler Nichols tuned PID controller and GA tuned PID controller. Tuned PID controller using HGNMA gives smaller overshoot and settling time compared to Ziegler Nichols and GA tuned PID controllers. The result proves that HGNMA has better performance than other two approaches with smaller settling time and overshoot.

\section{REFERENCE}

[1] G.F. Franklin, J.D. Powell and A. Emami-Naieni," Feedback Control of Dynamic Systems", Addison-Wesley, 1988

[2] K.J. Aström and T. Hägglund, "PID Controller: Theory, Design and Tuning", Instrument Society of America, North Carolina, 1995.

[3] Maolong Xi, Jun Sun and Wenbo Xu "Parameter optimization of PID controller based on quantum- behaved particle swarm optimization algorithm", DCDIS Series B, complex systems and applications, Vol. 14 (s2) pp 603-607, 2007.

[4] R. A. Krohling and J. P. Rey, "Design of optimal disturbance rejection PID controllers using genetic algorithm," IEEE Trans. Evol. Comput., vol. 5, pp. 7882, Feb. 2001.

[5] Shan Li, Jinhua Li., "IMC-PID Control of Ultra-Sonic Motor Servo System Based on Neural Network", Intelligent Control and Automation, WCICA. Page(s): 8275 - 8279, 2006.

[6] Qiao, W. and Mizumoto, M, 1996. " PID type fuzzy controller and parameters adaptive method", fuzzy sets and system. Page(s): 23-35.

[7] L. Davis, "Handbook of Genetic Algorithms", VNR Comp. Library, 1990. 
[8] Jin-Sung Kim et al, "Auto tuning PID controller based on improved genetic algorithm for reverse Osmosis", World Academy of Science, Engineering and Technology, 47/2008

[9] M. E. El-Telbany, "Employing Particle Swarm Optimizer and Genetic Algorithms for Optimal Tuning of PID Controllers: A Comparative Study" ICGST-ACSE Journal, Volume 7, Issue 2, November 2007

[10] Gaing, Z.-L., "A particle swarm optimization approach for optimum design of PID controller in AVR system", IEEE Transactions on EnergyConversion, vol. 19 , no. 2 , pp. $384-391,2004$.

[11] Marco A. Luersn, Rodolphe Le Riche, "Globalized Nelder-Mead Method for engineering optimization", European Journal of Computers and Structures, vol. 82, no 23-26, pp. 2251-2260, (2004).

[12] Rachid Chelouah and Patrick Siarry, "Genetic and Nelder-Mead algorithms hybridized for a more accurate global optimization of continuous multi- minima functions", European Journal of Operational Research pp. 335-348, (2003).

[13] A. Lasheen and et al, "Using hybrid genetic and Nelder-Mead algorithm for decoupling of MIMO systems with application on two coupled distillation columns process", International Journal Of Mathematics and Computers In Simulation, Issue 3, Volume 3, PP.146-157, 2009

[14] Hisn-Chuan Kuo, Jiang-Ren Chang and Kai-Shun Shyu; "A Hybrid Algorithm of evaluation and Simplex Methods Applied to Global Optimization"; Journal of Marine Science and Technology, Vol. 12-No. 4, pp. 280-289, (2004).

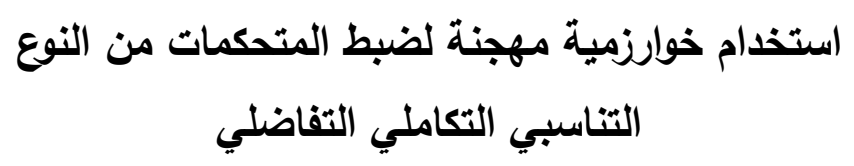

يقدم هذا البحث خوارزمية مهجّنة باستخدام كل من الخوارزمية الجينية وخوارزمية نيلدرميد لضبط متحكم من النوع التتاسبي التكاملي التفاضلي بهدف الوصوله باسنال إلى القيم المتلى لمعاملات التتاسب

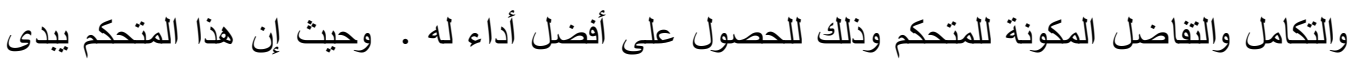
خصائص جيدة تشمل سهولة التطبيق والتقارب المستقر وكذلك كفاءة حسابية عالية فقد تم تتفيذ هذه الخوارزمية المهجّنة باستخدام الحاسب للوصول إلى قيم متغيرات المتحكم وبمقارنة النتائج التي نم الحصول عليها من جراء استخدام هذا الكود المطوّر بكل من النتائج الناتجة عن استخدام الخوارزمية الجينية فقط وكذلك تلك الناتجة عن تطبيق طريقة زيجلر - نيكلوس فإن الطريقة المقترحة أثنتت كفاءة

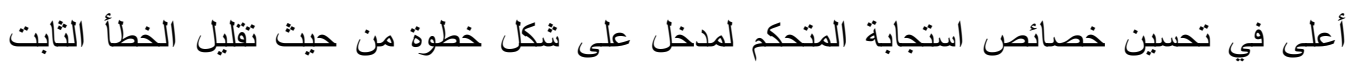
وزمن الصعود إلى حالة الاستقرار وزمن الاستقرار وكذلك أقصى إزاحة رأسية. 